\title{
The rise and fall of hospitalizations for skin infections in New Zealand, 2004-2014: trends by ethnicity and socioeconomic deprivation
}

\author{
A. $\operatorname{LIM}^{1 *}$, J. RUMBALL-SMITH ${ }^{2}$, R. JONES ${ }^{3}$ AND I. KAWACHI ${ }^{4}$ \\ ${ }^{1}$ Faculty of Medical and Health Sciences, University of Auckland, New Zealand \\ ${ }^{2}$ Population and Public Health, Northland District Health Board, New Zealand \\ ${ }^{3}$ Te Kupenga Hauora Māori, Faculty of Medical and Health Sciences, University of Auckland, New Zealand \\ ${ }^{4}$ Department of Social and Behavioral Sciences, Harvard School of Public Health, Boston, MA, USA
}

Received 24 July 2016; Final revision 7 October 2016; Accepted 26 October 2016;

first published online 1 December 2016

\section{SUMMARY}

Since the 1990s, New Zealand (NZ) has seen an increasing incidence of paediatric skin infections leading to hospitalization. We sought to describe the epidemiology of these infections over time. Hospital discharge data were analysed for all children admitted to any NZ public hospital from 2004 to 2014. We found the age-standardized incidence of hospitalizations due to skin infections increased during this period from 522/100 000 in 2004 to a peak of 644/100 000 in 2011, after which it declined to 524/100 000 in 2014. This decline in incidence was driven primarily by the decline in hospitalization rates in high-risk groups including children living in the most deprived areas as well as Māori and Pacific children. Recent targeted policies focused on improving healthcare access through school-based and primary care-based interventions in these high-risk groups could potentially explain the decline in hospitalization rates. However, even with the closing of the inequality gap, significant socioeconomic and ethnic health disparities persist.

Key words: Health policy, incidence, public health, skin infections.

\section{INTRODUCTION}

Bacterial skin infections are most commonly caused by Staphylococcus or Streptococcus species and include a number of conditions, such as cellulitis [1-4]. Best practice management of simple skin infections focuses on early primary-care review with either topical or oral antibiotic use [3-5]. Hospitalization for intravenous antibiotics and/or surgical debridement is reserved for children who have become systemically unwell from their skin infection [4]. For the purpose of this study, skin infection leading to hospitalization will represent serious skin infection.

\footnotetext{
* Author for correspondence to: Dr A. Lim, 1, Hirimate Place, Flat Bush, Auckland, New Zealand.

(Email: angela.lim@outlook.com)
}

In New Zealand (NZ), the rate of paediatric skin infections is unusually high compared to other Organization for Economic Co-operation and Development (OECD) countries and is a leading cause of hospitalization in children [4-6]. Since the 1990s, hospitalizations for skin infection have risen in $\mathrm{NZ}[2,4,5]$ and in the same period, ethnic and socioeconomic disparities in this patient group have also widened [2].

Serious skin infections disproportionately affect children aged $<5$ years, particularly children of Māori (indigenous population) or Pacific ethnicity, and those living in areas of high socioeconomic deprivation [2, 7-13], the latter also associated with longer hospital stays [11].

The factors driving this pattern of health inequality are multiple and complex [12]. Identified 
socioeconomic drivers associated with an increased risk of serious skin infections work via two common mechanisms. One is through increased disease incidence, such as overcrowded housing and poor health literacy, and the other due to delayed or inadequate treatment related to cost and barriers to accessing primary care $[2-4,8,14]$.

Building on the earlier work of O'Sullivan et al. [2], we sought to describe changes in disease distribution that may have occurred, particularly post-2007 when their time series concluded. We also aimed to investigate the contribution of socioeconomic deprivation to ethnic disparities in paediatric skin infection in NZ from 2004 to 2014.

\section{METHODS}

Hospital discharge data were obtained from the NZ Ministry of Health's National Minimum Dataset. Inclusion criteria for the study subjects were: children aged $0-14$ years who had at least one overnight admission at any NZ public hospital between 1 January 2004 and 31 December 2014 with a primary or secondary discharge diagnosis from a defined list of International Classification of Disease (ICD-10) codes for skin infections (see Supplementary Appendix, Table A1). This classification references previous international and NZ work [2]. Exclusion criteria were: overseas visitors, patients who were discharged the same day as their admission, patients with both cancer-related and Type 1 diabetes diagnostic codes who were likely to be immunocompromised $[15,16]$.

Each hospital discharge record has a unique patient identifier (encrypted National Health Index number). To avoid duplication of patient data due to re-admissions as well as between- or within-facility referrals for the same condition, analyses were restricted to the initial admission record for episodes with the same diagnostic code within a 30-day period.

Also provided in each record is the New Zealand Deprivation Index score (NZDep), which is based on each patient's residential domicile census area, with a median size of 2000 people. The NZDep is a well-validated method of describing small-area socioeconomic deprivation based on nine variables extracted from national census data $[2,17]$. Decile 1 indicates the least deprived $10 \%$ and decile 10 the most deprived $10 \%$ of areas in NZ [2, 17, 18]. This study used the 2006 version of NZDep index score.
Four major ethnic groups were used in this study: NZ European, Māori, Pacific and Asian. Pacific cover a range of ethnicity including, Samoan, Cook Island Maori, Fijian, Tongan, Niuean and Tokelauan. Ethnicity was identified by the caregivers with up to three ethnic group codes recorded for each patient. The dataset obtained uses an algorithm to prioritize ethnic group codes if more than one was present, where Māori ethnic identification is given the highest priority score followed by Pacific, Asian and finally NZ European. All 'Other' ethnicities were grouped together with the NZ European ethnic group, as they comprised only $1 \%$ of cases. From here on this group is categorized as NZ European.

Skin infection-related hospitalization rates were used to infer incidence of serious skin infection in NZ [2]. Age-specific incidence rates by gender, ethnicity and deprivation levels were calculated and hospitalization rates were calculated by dividing the age-specific counts of hospital admissions by the corresponding denominator populations for each census area unit. The denominator populations were obtained from the 2006 and 2013 NZ Censuses, using linear interpolation to provide estimates in the inter-census years; these rates were directly age-standardized using the World Health Organization world standard population data.

Trends over time and between subpopulations were explored by linear regression and calculation of rate ratios (RR) with 95\% confidence intervals (CI). Confidence intervals for yearly incidence rates were not calculated as this study was based on the entire eligible NZ paediatric population. Analyses were conducted using SPSS v. 22 (IBM Corp., USA).

\section{RESULTS}

A total of 74715 cases of skin infection hospitalizations met the inclusion criteria; 6391 were excluded and the remaining 68324 cases were analysed (see Supplementary Appendix, Fig. A2 for a breakdown of exclusions). Of these cases, $62 \cdot 7 \%$ were captured through primary diagnosis.

\section{Infection rates from 2004 to 2014}

During the 11-year period, the age-standardized incidence rates of serious skin infections generally increased annually from 2004 to 2011 . These infections increased from 522/100000 in 2004 to reach peak incidence in 2011 at $644 / 100000$ before 


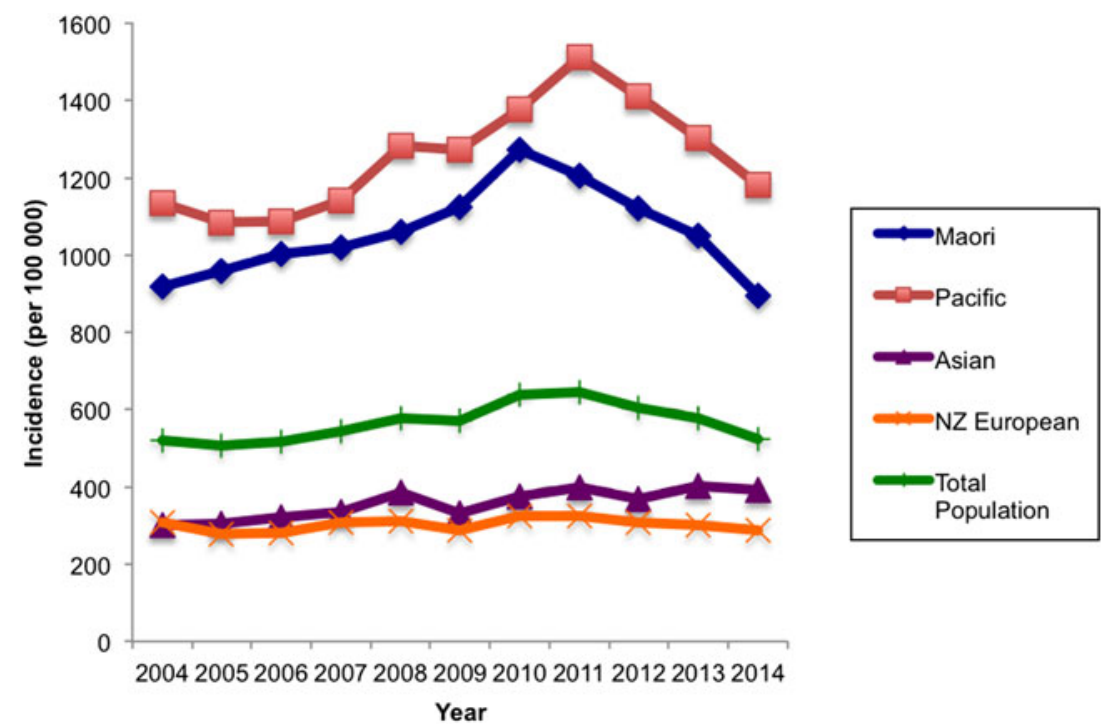

Fig. 1. Age-standardized incidence of skin infection-related hospitalization for children aged 0-14 years in New Zealand (NZ) from 2004 to 2014, and ethnic distribution.

decreasing to $524 / 100000$ in 2014, a level last seen in 2004 (Fig. 1). The post-2011 decline in hospitalization rates was statistically significant $(P$ for linear trend $<$ $0 \cdot 001)$.

\section{Gender, age and ethnicity}

Table 1 describes the serious skin infections in NZ children, from 2004 to 2014, according to demographic characteristics. Boys had a greater risk of infection (RR 1.42, 95\% CI 1.29-1.58) than girls, and the incidence of infections decreased with increasing age; age $0-4$ years (RR 3.05, 95\% CI 2.72-3.41) compared to age 10-14 years. This difference remained constant over the study period (Fig. 2).

Throughout the study period, serious skin infections were significantly higher in Māori and Pacific children compared to the other ethnic groups (Fig. 1 and Table 1). Pacific children had the greatest risk of hospitalization (RR 4.17, 95\% CI 3.68-4.72) compared to NZ European, closely followed by Māori (RR 3.51, 95\% CI 3.09-3.98) children. Between 2004 and 2011, the disparity in hospitalization rates continued to widen for Māori, Pacific and Asian children compared to NZ European children. Māori children reached a peak RR of 3.9 in 2010, and Pacific children a RR of 4.7 in 2011 . The ethnic inequality subsequently narrowed between 2012 and 2014, due to the hospitalization rates falling more quickly, in both absolute and relative terms, for Māori and Pacific compared to NZ European children (Fig. 1).
Linear regression showed the decline to be statistically significant $(P<0 \cdot 001)$.

\section{Socioeconomic deprivation}

As expected, the incidence of serious skin infection was lowest in areas with the least deprivation, with incidence increasing in parallel with deprivation (see Table 1). Children living in NZDep decile 10 (most deprived) had a RR of $5 \cdot 81$ (95\% CI 5.58-6.05) compared to those in NZDep decile 1 (least deprived). Among those living in NZDep decile 10, the incidence rates for Māori children were $2 \cdot 1$ times $(95 \%$ CI $2 \cdot 01$ $2 \cdot 23$ ) those of NZ European children within the same deprivation stratum. Incidence rates for Pacific children were $2 \cdot 4$ times $(95 \%$ CI $2 \cdot 31-2 \cdot 57)$ higher than those of NZ European children (Fig. 3).

Figure 3 also shows that skin infection incidence exhibited a steeper socioeconomic gradient in Māori and Pacific children compared to NZ European and Asian children. For Māori children, the risk of hospitalization was 4.9 times $(95 \%$ CI $4 \cdot 34-5 \cdot 5)$ higher for those living in the most deprived areas compared to their counterparts in the least deprived areas. While for NZ European children, those living in the most deprived areas had $3 \cdot 1$ times (95\% CI $2 \cdot 92-3 \cdot 32)$ greater risk than those in the least deprived areas. This figure shows the absolute risk difference is greater for NZDep within each ethnicity compared to the absolute risk difference between ethnicity within any level of NZDep. 
Table 1. Paediatric serious skin infection frequency, incidence and rate ratio by gender, age group, ethnicity and deprivation, 2004-2014.

\begin{tabular}{llll}
\hline \hline Variable & $f$ & Incidence* & RR $(95 \%$ CI $)$ \\
\hline Gender & & & \\
$\quad$ Female & 29445 & 617 & $1 \dagger$ \\
$\quad$ Male & 38879 & 879 & $1 \cdot 42(1 \cdot 29-1 \cdot 58)$ \\
Age group, years & & & \\
10-14 & 13182 & 395 & $1 \dagger$ \\
$5-9$ & 15731 & 483 & $1 \cdot 22(1 \cdot 07-1 \cdot 4)$ \\
$0-4$ & 39411 & 1203 & $3 \cdot 05(2 \cdot 72-3 \cdot 41)$ \\
Ethnicity & & & \\
NZ European & 21803 & 302 & $1 \dagger$ \\
Asian & 3775 & 360 & $1 \cdot 19(1 \cdot 02-1 \cdot 39)$ \\
Māori & 26411 & 1059 & $3 \cdot 51(3 \cdot 09-3 \cdot 98)$ \\
Pacific & 16335 & 1258 & $4 \cdot 17(3 \cdot 68-4 \cdot 72)$ \\
NZDepł & & & \\
1 & 2680 & 276 & $1 \dagger$ \\
2 & 3264 & 352 & $1 \cdot 28(1 \cdot 21-1 \cdot 34)$ \\
3 & 3201 & 359 & $1 \cdot 30(1 \cdot 24-1 \cdot 37)$ \\
4 & 3496 & 399 & $1 \cdot 45(1 \cdot 38-1 \cdot 52)$ \\
5 & 4433 & 516 & $1 \cdot 87(1 \cdot 78-1 \cdot 96)$ \\
6 & 5070 & 594 & $2 \cdot 15(2 \cdot 05-2 \cdot 26)$ \\
7 & 6408 & 757 & $2 \cdot 74(2 \cdot 62-2 \cdot 87)$ \\
8 & 8959 & 1018 & $3 \cdot 69(3 \cdot 53-3 \cdot 85)$ \\
9 & 11983 & 1225 & $4 \cdot 44(4 \cdot 26-4 \cdot 63)$ \\
10 & 18793 & 1604 & $5 \cdot 81(5 \cdot 58-6 \cdot 05)$ \\
\hline \hline RR Rate & & &
\end{tabular}

$\mathrm{RR}$, Rate ratio; CI, confidence interval.

$f$, Frequency is number of cases in 2004-2014.

* Average annual incidence/100 000 based on usually resident population (from NZ Census).

$\dagger$ Arbitrary reference category.

\$ The New Zealand Deprivation Index (NZDep) is a measure of socioeconomic deprivation based on nine variables extracted from census data. NZDep 1 indicates least deprivation and 10 indicates highest deprivation.

The annual rate ratio of children living in NZDep decile 10 areas compared to NZDep decile 1 remained relatively stable from 2004 to 2011, with a RR between 6 and 7 (Fig. 4). However from 2011 onwards, the rate ratios declined from 6.0 in 2011 to 4.3 in 2014 . This trend was primarily driven by the absolute declines in hospitalization rates for the most deprived areas. Linear regression showed the decline to be statistically significant $(P<0 \cdot 001)$.

\section{DISCUSSION}

The incidence of hospitalizations for skin infection, as well as ethnic and socioeconomic disparities in skin infection, were previously reported to have increased from 1990 to 2007 [2, 6-10]. Here, we have extended a previously published time series [2] to examine changes in the trends of these infections. Following a peak incidence in 2011, we found a reversal in this trend, with a return to levels previously seen in 2004. Furthermore, the high-risk groups have remained unchanged over time and include boys, children aged $<5$ years, Māori and Pacific children, and those living in areas of high socioeconomic deprivation [2, 6-10]. Higher hospitalization rates in boys and those aged $<5$ years could potentially be due to higher incidence of injuries and/or lower hospital admission thresholds [2],

A significant change from the latter study was the closing of ethnic and socioeconomic disparities, which drove the overall decline in hospitalization rates and reduced the outcome gap $[19,20]$. In populations with higher socioeconomic deprivation, the spread of skin infection mainly occurs through greater skin-skin contact from children living in overcrowded housing conditions $[2-4,8]$. Poor health literacy, which tends to be more common in these populations, can also play a part, leading to inadequate prevention of the spread of disease as well as lower levels of medication adherence $[2-4,8,21]$.

For those in disadvantaged communities, the higher incidence of severe skin infection requiring hospitalbased interventions is in part attributable to barriers to accessing primary healthcare. These include the costs of accessing timely treatment [2, 8, 14, 22-24], such as those associated with general practitioner copayments and the purchase of prescribed antibiotics $[2,8,14,22-24]$. There are also indirect costs such as travel time to the clinic, conflicts in work schedules and challenges in arranging for transport and childcare [22-24].

The descriptive data presented here are limited in their capacity to explain the reduction in health inequalities. However, the factors that drive hospitalization highlight areas for targeted interventions that could serve as potential explanations for the observed changes. Although we lack direct evidence, several skin infection-related interventions were implemented in NZ around the 2011 time period that might have contributed to the decline in hospitalization rates but owing to the multifactorial nature of this condition's aetiology it is difficult to attribute causation to any one specific intervention. These interventions included the Ministry of Health's school-based rheumatic fever clinics delivered in 11 of the 20 District Health Boards (DHBs) $[25,26]$. DHBs are publicly funded entities that serve a defined geographical area to provide 


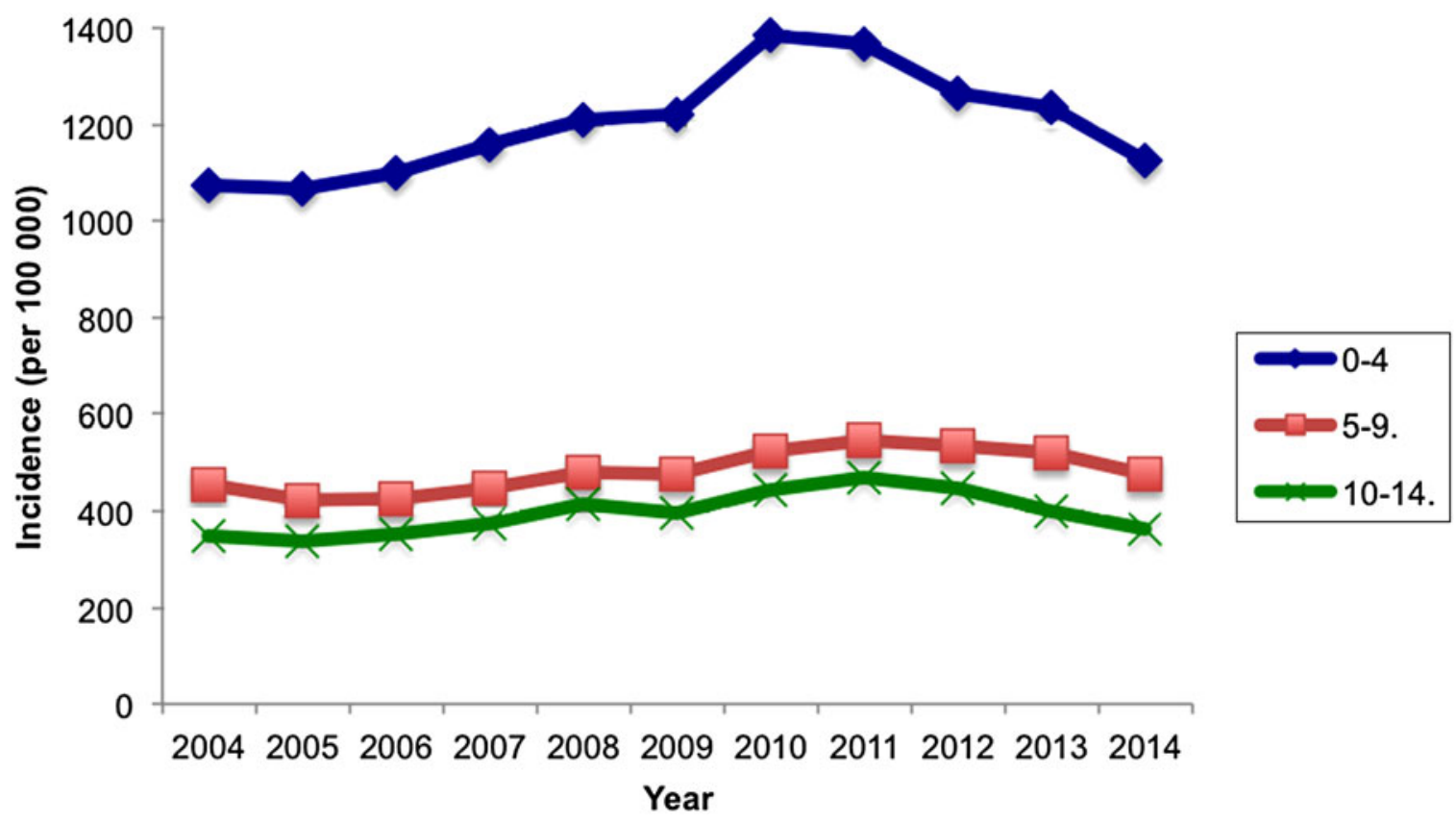

Fig. 2. Age-standardized incidence of skin infection-related hospitalization for children in three different age groups from 2004 to 2014.

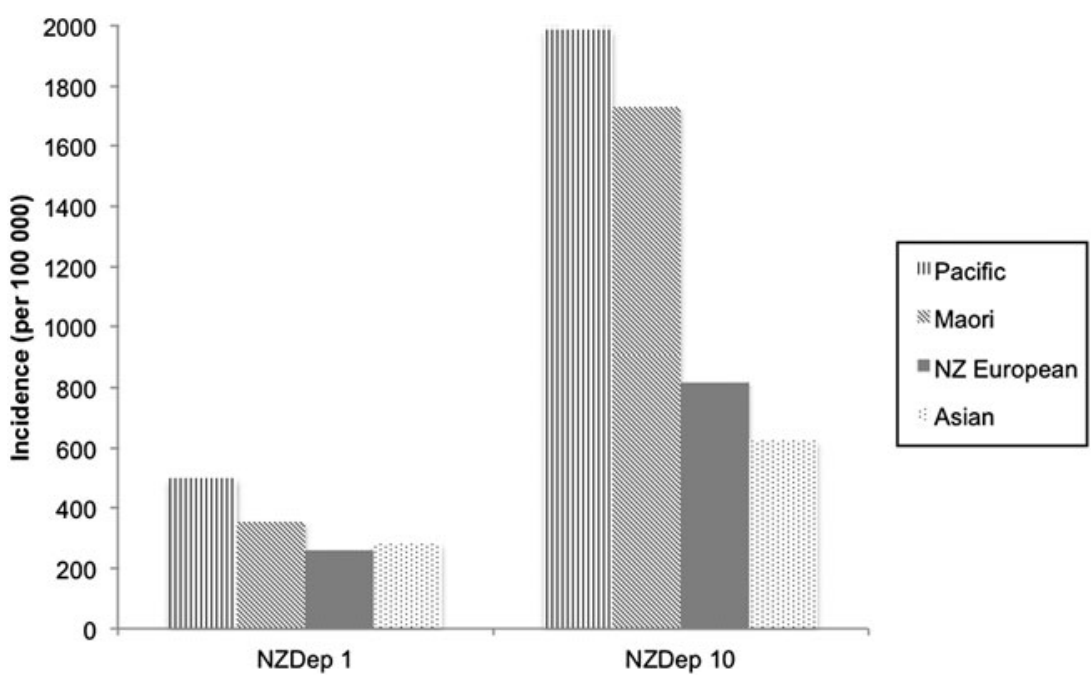

Fig. 3. Age-standardized incidence of skin infection-related hospitalization, comparing different ethnic groups according to social deprivation scale (NZDep decile 1 and NZDep decile 10).

free and/or subsidized health services. Within those 11 DHBs, more than half also included a targeted skin infection intervention programme, which comprised a free diagnostic assessment of infection and free provision of antibiotics. Also bundled in this programme was education regarding skin infection to the child and their family with the aim of improving health literacy. This intervention was targeted at schools with a high proportion of Māori and Pacific children living in areas of high socioeconomic deprivation $[25,26]$.
International reviews of health promotion programmes identify schools as an effective setting to address the risk factors of diseases, as they are able to reach large numbers of children directly [26, 27]. School-based interventions also create structurallevel conditions that facilitate access to healthcare professionals and antibiotics, and directly address ethnic and socioeconomic disparities in accessing care [27]. Primary-care-based interventions introduced in NZ include Primary Options for Acute Care, which 


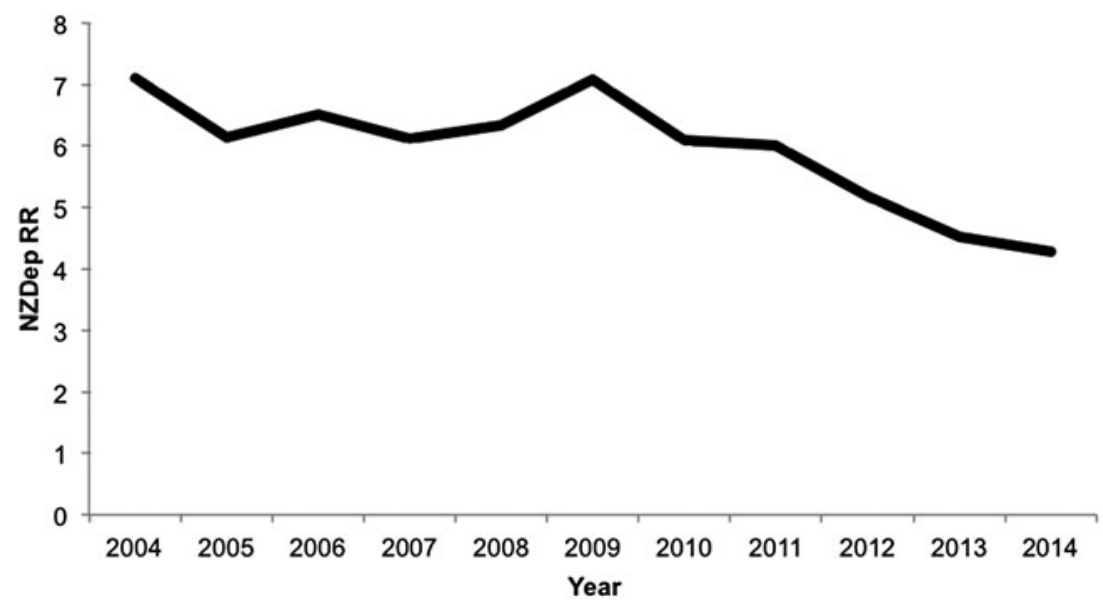

Fig. 4. Rate ratio (RR) of skin infection-related hospitalization, comparing NZDep decile 10 to NZDep decile 1.

allow children with cellulitis to receive intravenous antibiotics in a primary-care setting, who would otherwise have been managed in hospital [28]. This was accompanied by an improvement in primary-care staff access to best practice paediatric skin infection guidelines. Further evaluation and research on these interventions is required before it is possible to determine the extent to which any of them contributed to the decline in incidence observed. Notably, as hospitalization rates have also fallen in non-targeted population groups, these interventions alone cannot explain the decline in skin infection hospitalization rates.

Regardless of the closing of the gap, there remain substantial inequalities in the overall rates of hospitalization for Māori and Pacific children compared to their NZ European counterparts. This is illustrated by the persistence of the gradient gap, whereby the risk of hospitalization is compounded for Māori and Pacific children with increasing levels of deprivation $[2,6-10,20]$. The deprivation-specific data suggest that it is more than just socioeconomic deprivation driving the ethnic inequalities and other factors such as persistent racial discrimination need to be considered [19, 29, 30]. For Māori in particular, social inequities also need to be considered in the context of historical and contemporary colonization involving dispossession of social, cultural, political and economic resources $[2,19,30]$ in order to make progress towards reducing or eliminating such disparities.

This is the only study we are aware of that has conducted ethnic-specific deprivation analyses as well as describing current interventions that could potentially explain changes in skin infection incidence. Challenges in tackling health inequality along ethnic and socioeconomic lines are not unique to NZ and our aim was to shed light on key areas for the international community to consider. However, the study has some limitations. First, the dataset was subject to DHB-specific variations that may have existed in regards to admission criteria to hospital, and treatment and coding criteria. We sought to account for the discrepancy in administrative records by excluding patients who were discharged on the same day as admission. Second, the dataset did not include more in-depth clinical information such as the bacteriological findings, the severity of infection, and treatments received. Third, by utilizing the NZDep index of deprivation, it was not possible to make individual-level attribution regarding socioeconomic deprivation. Last, the effects of seasonality and levels of rurality in serious skin infection, as previously reported by others [2], were not explored.

Future studies could include a multi-level analysis to measure the interaction between ethnicity and socioeconomic deprivation levels when predicting outcomes such as skin infection hospitalization, length of stay and re-admission rates.

\section{SUPPLEMENTARY MATERIAL}

For supplementary material accompanying this paper visit https://doi.org/10.1017/S0950268816002685.

\section{ACKNOWLEDGEMENTS}

The authors gratefully acknowledge the statistical advice and time given by Rob McNeill, Mike Picot and Randall Cork. This research received no specific grant from any funding agency, commercial or notfor-profit sectors. 


\section{DECLARATION OF INTEREST}

None.

\section{REFERENCES}

1. Stulberg D, Penrod M, Blatny R. Common bacterial skin infections. American Family Physician 2002; 66: 119-124.

2. O'Sullivan C, Baker M, Zhang J. Increasing hospitalisations for serious skin infections in New Zealand children, 1990-2007. Epidemiology and Infection 2011; 139: 1794-1804.

3. Bernard P. Management of common bacterial infections of the skin. Current Opinion in Infectious Diseases 2008; 21: $122-128$.

4. Ete-Rasch E, Nelson K. Management of skin infections in pacific children prior to hospitalisation. Journal of Primary Health Care 2013; 5: 43-51.

5. O'Sullivan C, Baker M. Skin infections in children in a New Zealand primary care setting: exploring beneath the tip of the iceberg. New Zealand Medical Journal 2012; 125: 70-79.

6. Baker M, et al. Increasing incidence of serious infectious diseases and inequalities in New Zealand: a national epidemiological study. Lancet 2012; 379: 1112-1119.

7. Williamson D, et al. Increasing incidence and sociodemographic variation in community-onset Staphylococcus aureus skin and soft tissue infections in New Zealand children. Pediatric Infectious Disease Journal 2013; 32: 923-925.

8. Bailie R, et al. Skin infection, housing and social circumstances in children living in remote Indigenous communities: testing conceptual and methodological approaches. Biomed Central Public Health 2005; 5: 128.

9. O'Sullivan C, Baker M. Serious skin infections in children: a review of admissions to Gisborne Hospital (2006-2007). New Zealand Medical Journal 2012; 125: $55-69$.

10. O'Sullivan C, et al. The epidemiology of serious skin infections in New Zealand children: comparing the Tairawhiti region with national trends. New Zealand Medical Journal 2012; 125: 40-54.

11. Petrou S, Kupek E. Socioeconomic differences in childhood hospital inpatient service utilisation and costs: prospective cohort study. Journal of Epidemiology and Community Health 2005; 59: 591-597.

12. Berkman L, Kawachi I, Glymour M. Social Epidemiology, 2nd edn. New York: Oxford University Press, 2014, pp. 17-32.

13. Phelan J, et al. 'Fundamental causes' of social inequalities in mortality: a test of the theory. Journal of Health and Social Behavior 2004; 45: 265-285.

14. Brabyn L, Barnett R. Population need and geographical access to general practitioners in rural New Zealand. New Zealand Medical Journal 2004; 117: 41-54.
15. Roman E, et al. Childhood acute lymphoblastic leukemia and infections in the first year of life: a report from the United Kingdom childhood cancer study. American Journal of Epidemiology 2006; 165: 496-504.

16. Atkinson M, Eisenbarth G. Type 1 Diabetes: new perspectives on disease pathogenesis and treatment. Lancet 2001; 358: 221-229.

17. Socioeconomic Deprivation Indexes. NZDep and NZiDep, Department of Public Health (http://www. otago.ac.nz/wellington/departments/publichealth/research/ hirp/otago 020 194.html). Accessed 25 April 2016.

18. McFadden K, et al. Socioeconomic deprivation and the incidence of cervical cancer in New Zealand: 19881998. New Zealand Medical Journal 2004; 117: 1-8.

19. Bécares L, Cormack D, Harris R. Ethnic density and area deprivation: neighbourhood effects on Māori health and racial discrimination in Aotearoa/New Zealand. Social Science and Medicine 2013; 88: 76-82.

20. Reid P, Robson B, Jones C. Disparities in health: common myths and uncommon truths. Pacific Health Dialogue 2000; 7: 38-47.

21. Baguley D, et al. Prescribing for children - taste and palatability affect adherence to antibiotics: a review. Archives of Disease in Childhood 2012; 97: 293-297.

22. Early G, Seifried S. Risk factors for community-associated Staphylococcus aureus skin infection in children of Maui. Hawaii Journal of Medicine and Public Health 2012; 71: 218-223.

23. Fiscella K, et al. Inequality in quality: addressing socioeconomic, racial and ethnic disparities in health care. Journal of the American Medical Association 2000; 283: 2579-2584.

24. Snell-Johns J, Smith B, Mendez J. Evidence-based solutions for overcoming access barriers, decreasing attrition, and promoting change with underserved families. Journal of Family Psychology 2004; 18: 19-35.

25. RFPP Strategies and Initiatives. (http://www.health. govt.nz/our-work/diseases-and-conditions/rheumatic-fever/ rfpp-strategies-and-initiatives). Accessed 20 April 2016.

26. Vogel A, et al. Registered nurse assessment and treatment of skin sepsis in New Zealand schools: the development of protocols. New Zealand Medical Journal 2013; 126: 15-26.

27. Jackson S, et al. Integrated health promotion strategies: a contribution to tackling current and future health challenges. Health Promotion International 2007; 21: 75-83.

28. Primary Options for Acute Care. Management of cellulitis. (http://primaryoptions.co.nz/site_files/359/upload_ files/Primary_Optio12.pdf). Accessed 25 April 2016.

29. Sabin J, Rivara F, Greenwald A. Physician implicit attitudes and stereotypes about race and quality of medical care. Medical Care 2008; 46: 678-685.

30. Harris R, et al. Effects of self-reported racial discrimination and deprivation on Māori health and inequalities in New Zealand: cross-sectional study. Lancet 2006; 367: 2005-2009. 